\title{
Throughput Analysis for Cooperation in Random Access Networks
}

\author{
Amr A. El-Sherif ${ }^{1}$ and K. J. Ray Liu ${ }^{2}$ \\ ${ }^{1}$ Department of Electrical Engineering, \\ Alexandria University, Alexandria, 21544, Egypt. \\ amr.elsherif@ieee.org \\ ${ }^{2}$ Department of Electrical and Computer Engineering, \\ University of Maryland, College Park, MD 20742, USA. \\ kjrliu@umd.edu
}

\begin{abstract}
This paper aims at answering the questions of how to enable cooperative communications in random access networks. And, since cooperation introduces extra transmissions in the channel, what are the benefits and possible tradeoffs associated with cooperation? To achieve this goal, a novel cooperative protocol for wireless networks based on IEEE 802.11 random access protocol is proposed. The proposed protocol guarantees an uncontested channel access to the relay. Interaction between different network nodes is characterized and performance analysis is conducted through Markov chain models in conjunction with queueing theoretical analysis. Results demonstrate significant gains in terms of throughput and delay performance compared with the non cooperative CSMA/CA protocol.
\end{abstract}

\section{INTRODUCTION}

Some works have focused on the impact and implementation of cooperative communications at higher network layers [1], [2], [3], [4]. Cooperation in random access networks has been considered in [3], where a distributed version of network diversity multiple-access (NDMA) [4] protocol was proposed. The main focus in [3] and [4] is on collision resolution. After the detection of a collision, a cooperative phase begins, in which retransmissions of the corrupted packets take place. Then, using conventional source separation techniques at the destination, the corrupted packets are retrieved.

In this work, we investigate the problem of enabling cooperation in wireless random access networks based on the IEEE 802.11 protocol. We consider a single hop network with multiple nodes and a single cooperative relay. In the CSMA/CA protocol, after a packet is transmitted over the channel, and irrespective of the outcome of this transmission, all network nodes will have to wait for a constant plus a random amount of time before making a transmission attempt. By allowing the relay to deviate from this access mechanism, and to access the channel immediately after each transmission attempt on the channel, the relay is guaranteed to have an uncontested access to the wireless medium, and there will be no danger of increasing the collision probability.

Based on the above description of the network operation, all nodes's queues are interacting, i.e., the service process of a given queue depends on the state of all other queues (whether they are empty or not). Two coupled Markov models are used to describe the operation of the relay and other network nodes. Both Markov models together are able to completely describe the dynamics of the network and interactions between different nodes. Moreover, queuing analysis is used to analyze the delay performance of the network. The results presented reveal significant gains in terms of network throughput, delay, and the number of supported nodes, due to cooperation and our proposed protocol. Furthermore, it is shown that, by virtue of the protocol design, collision probability has decreased rather than the expected increase due to extra transmissions on the channel.

\section{Channel AND System Models}

\section{A. Channel model}

We consider a Rayleigh fading channel model, the signal received at the access point or the relay is modeled as

$$
y_{i j}=\sqrt{G r_{i j}^{-\gamma}} h_{i j} x+\eta_{j}
$$

where $i$ is the source index, $j \in\{A, R\}$ is the access point or the relay index, $x$ is the transmitted signal, $G$ is the transmission power, assumed to be the same for all nodes, $r_{i} j$ denotes the distance between source node $i$ and its destination $j, \gamma$ is the path loss exponent, $h_{i} j$ the channel fading coefficients, modeled as zero-mean complex Gaussian random variables with unit variance, and $\eta_{j}$ is an additive noise term at the destination, modeled as zero-mean complex Gaussian random variable with variance $N_{0}$. We assume that the channel coefficients are constant for the duration of the transmission of one packet. In this work, without loss of generality, we only considered the case of a symmetric network, where all the inter-users channels are assumed to be statistically identical.

Success and failure of packet reception is characterized by outage events and outage probabilities. The outage probability is defined as the probability that the Signal to Noise Ratio (SNR) at the receiver is less than a given SNR threshold $\beta$. For the channel model in (1) the probability of outage can be written as,

$$
P_{i j}^{\text {out }}=\operatorname{Pr}\left\{\left|h_{i j}\right|^{2}<\frac{\beta N_{0} r_{i j}^{\gamma}}{G}\right\}=1-\exp \left(-\frac{\beta N_{0} r_{i j}^{\gamma}}{G}\right) .
$$

\section{B. IEEE 802.11 Operation}

Fig. 1 illustrates the operation of the IEEE 802.11 [5] basic access mechanism based on the CSMA/CA (Carrier Sense Multiple Access with Collision Avoidance) with binary slotted exponential backoff. A node with a new packet to transmit invokes the carrier sense mechanism. If the channel is idle for a period of time equal to a DIFS (Distributed Inter-Frame Space), the node transmits. Otherwise, if the 


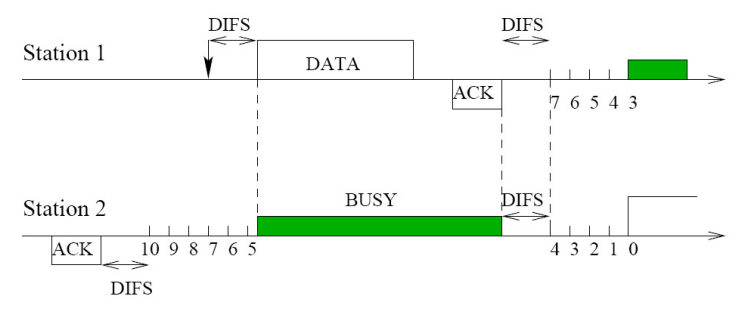

Fig. 1. DCF basic access mechanism; numbers in figure represent node's backoff timer.

channel is sensed busy the node persists to monitor the channel until it is measured idle for a DIFS. The node will then generate a random backoff interval before transmitting. The time immediately following an idle DIFS is slotted, and a node is allowed to transmit only at the beginning of each slot time.

The destination transmits an ACK upon a successful reception of a data frame. The ACK is immediately transmitted at the end of the packet, after a period of time called short interframe space (SIFS). As the SIFS is shorter than a DIFS, no other station is able to detect the channel idle for a DIFS until the end of the ACK. If the source node does not receive the ACK within a specified ACK_Timeout, the frame is assumed to be lost and the source node schedules the retransmission according to the backoff rule.

The random backoff interval is uniformly chosen in the range $(0, w-1)$. The value $w$ is called the contention window, and depends on the number of transmissions failed for the packet. At the first transmission attempt, $w$ is set equal to a minimum contention window value $\mathrm{CW}_{\min }$. After each unsuccessful transmission, $w$ is doubled, up to a maximum value $\mathrm{CW}_{\max }=2^{m} \mathrm{CW}_{\text {min }}$. The backoff timer is decremented as long as the channel is sensed idle, frozen when a transmission is detected on the channel, and reactivated when the channel is sensed idle again for more than a DIFS. The node transmits when the backoff time reaches zero.

\section{RANDOM ACCESS COOPERATION PROTOCOL}

In a wireless network nodes are unable to detect collisions by hearing their own transmissions. Therefore, there is no means to differentiate between a packet loss due to a collision from a packet loss due to wireless channel impairments. Hence, in case of an error contention window is doubled and a random amount of time is waited before a retransmission attempt. In a non-congested network, this results in an unnecessary increase in the delay and lower achievable throughput in[6], [7].

Our goal in this paper is to exploit spatial diversity offered by a cooperative relay node to mitigate the negative effects of channel fading on the system performance. In case the relay successfully receives a packet, but the AP does not receive that packet (relay makes use of the AP's ACK packet to know if a packet is successfully received by the AP), the relay stores that packet in its queue for transmission and sends an ACK packet over the channel to inform other nodes that the packet was received successfully. Upon receiving the relay's ACK packet, the node owning the packet will drop it from its queue and the delivery of that packet is now the relay's responsibility. Because of the relay's ACK packet, the node with a lost packet will not assume that the channel is congested and instead of doubling its contention window it will reset it to $C W_{\min }$.

In order not to increase the number of collisions and hence rendering its existence useless, the relay accesses the medium according to the following protocol:

- Following any transmission attempt (and irrespective of its outcome), the relay node attempts to transmit the packet at the head of its queue immediately after the AP ACK or the ACK_Timeout

- Moreover, the relay maintains a single stage backoff counter with contention window size $C W_{r}$

- When the relay's backoff counter reaches zero, it will attempt to transmit the packet at the head of its queue like other network nodes

- The relay will invoke the backoff procedure after each transmission attempt

By accessing the channel after each transmission attempt on the channel, the relay have the ability to serve the packets in its queue without causing any collisions. Furthermore, the relay's single backoff stage guarantees that the relay will have access to the channel even when all other nodes' queues are empty (hence, no transmission attempts take place).

\section{PERFormance AnAlysis}

\section{A. Markov Models and Analysis}

To analyze the performance of the proposed cooperative protocol, we start from the discrete-time Markov model for non-saturated sources developed in [8], and incorporate the channel effects and relay operation into the model. We consider two separate Markov chains, the first chain models source nodes while the second models the relay node.

We assume that the network consists of $N$ contending nodes in addition to the relay node. Each node has an infinite length queue to store packets. Each node receives packets from upper layer based on a Poisson arrival process with arrival rate $\lambda_{s}$ packets/sec (The super(sub)script s or $r$ are used to differentiate between source node and relay parameters), and fixed packet size $L$. The queuing model used will be discussed in details in section IV-B.

1) Source Nodes: Fig. 2 represents the discrete-time Markov chain modeling the operation of source nodes. Each node is modeled by a pair of integers $(i, k)$, states with a subscript $e$ model a node when its queue is empty. The backoff stage $i$, starts at 0 at the first attempt to transmit a packet and is increased by 1 every time a transmission attempt fails, up to a maximum value $m$. It is reset after a successful transmission. At any backoff stage $i \in[0, m]$, the backoff counter, $k$, is initially chosen uniformly between $\left[0, W_{i}^{s}-1\right]$, where $W_{i}^{s}=2^{i} W_{0}^{s}, \quad 0 \leq i \leq m$, is the range of the counter, and $W_{0}^{s}$ is the parameter $\mathrm{CW}_{\min }$ specified in the IEEE 802.11 


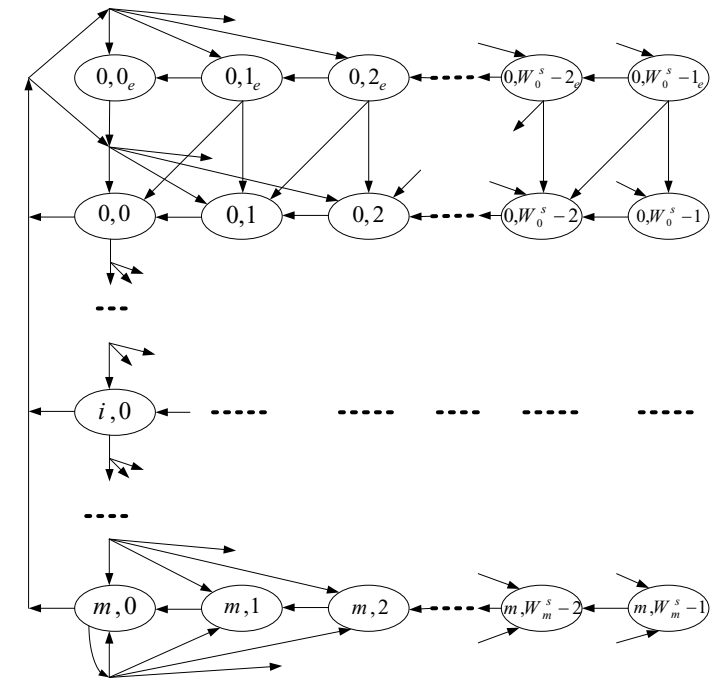

Fig. 2. Source node's Markov model.

standard. The backoff counter is decremented by 1 in each idle time slot of duration $\sigma$, and the node transmits when the backoff counter $k=0$.

The fundamental assumption in our model is that, at each transmission attempt, and regardless of the number of retransmissions suffered, each packet fails with a constant and independent probability $P_{f}^{s}$ or $P_{f}^{r}$, for the source nodes or relay node, respectively [8].

Let $\tau_{s}$ and $\tau_{r}$ be the probability that a source node or the relay transmit in a given slot, respectively. Now we are ready to write the Markov chain's transition probabilities, for $0 \leq$ $i \leq m$

$$
\begin{array}{ll}
P\{(i, k) \mid(i, k+1)\}=P_{i}, & 0 \leq k \leq W_{i}^{s}-2 \\
P\{(0, k) \mid(i, 0)\}=\frac{\left(1-q_{s}\right)\left(1-P_{f}^{s}\right)}{W_{0}^{s}}, & 0 \leq k \leq W_{0}^{s}-1 \\
P\left\{(0, k)_{e} \mid(i, 0)\right\}=\frac{q_{s}\left(1-P_{f}^{s}\right)}{W_{0}^{s}}, & 0 \leq k \leq W_{0}^{s}-1 \\
P\{(i, k) \mid(i-1,0)\}=P_{f}^{s} / W_{i}^{s}, & \\
P\{(m, k) \mid(m, 0)\}=P_{f}^{s} / W_{m}^{s}, &
\end{array}
$$

where $q_{s}$ is the probability that the node's queue is empty upon a departure (see section IV-B). $P_{i}$ is the probability that the channel is sensed idle by the source node (i.e., all the remaining $N-1$ source nodes and the relay node are not attempting to transmit), and is given by $P_{i}=\left(1-\tau_{s}\right)^{N-1}(1-$ $\left.\tau_{r}\right)$. The probability of a failed transmission attempt is given by

$$
\begin{aligned}
P_{f}^{s} & =1-\left(1-\tau_{s}\right)^{N-1}\left(1-\tau_{r}\right)\left(\left(1-P_{s A}^{\text {out }}\right)\right. \\
& \left.+\left(1-P_{s R}^{\text {out }}\right)-\left(1-P_{s A}^{\text {out }}\right)\left(1-P_{s R}^{\text {out }}\right)\right),
\end{aligned}
$$

which is the complement of the probability of the event that there is no collision, and that either the access point or the relay have correctly received the packet.
Given that the node's queue is empty and the chain is in state $(0, k)_{e}$, in case of a packet arrival, the backoff counter is decremented and the chain makes a transition into the $(0, k-1)$ state, otherwise, the chain transits into $\left(0,(k-1)_{e}\right)$. When the backoff timer reaches zero, the node remains in state $\left(0,0_{e}\right)$ as long as the queue is empty. If a packet arrives, then the node moves into state $(0, k)$, where $k$ is uniformly chosen in the range $\left[0, W_{0}^{s}-1\right]$. Therefore we have

$$
\begin{array}{ll}
P\left\{(0, k)_{e} \mid 0,(k+1)_{e}\right\}=P_{i}\left(1-a_{i}\right), & 0 \leq k \leq W_{0}^{s}-2 \\
P\left\{(0, k) \mid\left(0,(k+1)_{e}\right)\right\}=P_{i} a_{i}, & 0 \leq k \leq W_{0}^{s}-2 \\
P\left\{(0, k) \mid\left(0,0_{e}\right)\right\}=\left(1-P_{i}\right) a_{b}, & 0 \leq k \leq W_{0}^{s}-1
\end{array}
$$

where $a_{i}$ and $a_{b}$ are the probabilities of at least one packet arrival during an idle or a busy slot, respectively. From the Poisson arrival assumption, these probabilities ar given by $a_{i}=1-e^{-\lambda_{s} \sigma}$ and $a_{b}=1-e^{-\lambda_{s} T_{b}}$, where $\sigma$ is the idle slot duration, and $T_{b}$ the busy slot duration (for simplicity we neglect the difference in durations between successful and unsuccessful transmission attempts). Typically, $\sigma=20 \mu \mathrm{s}$, and $T_{b}=2160.4 \mu \mathrm{s}$, based on $11 \mathrm{Mbps}$ channel rate and packet size $L=2312$ octets [5].

Let $\pi_{s}(i, k)$ denote the stationary probability of being in state $(i, k)$. To solve for the stationary distribution of this Markov chain, we will find expressions for all the stationary probabilities as a function of $\pi_{s}(0,0)$. First, we will find expressions for all the stationary probabilities as a function of $\pi_{s}(0,0)$. We will omit the derivations details for the lack of space and just write down the resulting expressions,

$$
\begin{aligned}
\pi_{s}(i, 0) & =\left(P_{f}^{s}\right)^{i} \pi_{s}(0,0), \quad 0<i<m \\
\pi_{s}(m, 0)= & \frac{\left(P_{f}^{s}\right)^{m}}{1-P_{f}^{s}} \pi(0,0), \\
\pi_{s}(i, k)= & \frac{W_{i}^{s}-k}{P_{i} W_{i}^{s}} \pi_{s}(i, 0), \\
\pi_{s}(0, k)_{e}= & \pi_{s}(0,0) \frac{q_{s}}{W_{0}^{s}\left(P_{i}+\left(1-P_{i}\right) a_{b}\right)} \frac{1-c^{W_{0}^{s}-k}}{1-c}, \\
\pi_{s}(0,0)_{e}= & \pi_{s}(0,0) \frac{q_{s}}{W_{0}^{s}\left(P_{i} a_{i}+\left(1-P_{i}\right) a_{b}\right)} \\
& \cdot\left(1+\frac{P_{i}\left(1-a_{i}\right)}{P_{i}+\left(1-P_{i}\right) a_{b}} \frac{1-c^{W_{0}^{s}-1}}{1-c}\right) \\
\pi_{s}(0, k)= & \frac{W_{0}^{s}-k}{P_{i} W_{0}^{s}}\left[\left(1-q_{s}\right) \pi_{s}(0,0)\right. \\
+ & \left.\left(\left(1-P_{i}\right) a_{b}+P_{i} a_{i}\right) \pi_{s}(0,0)_{e}\right] \\
& +\sum_{l=k+1}^{W_{0}^{s}-1}\left(P_{i} a_{i}+\left(1-P_{i}\right) a_{b}\right) \pi_{s}(0, l)_{e}+\left(1-P_{i}\right) a_{b}
\end{aligned}
$$

where $c=\frac{P_{i}\left(1-a_{i}\right)}{P_{i}+\left(1-P_{i}\right) a_{b}}$.

Imposing the normalization condition $\sum_{i=0}^{m} \sum_{k=0}^{W_{i}^{s}-1} \pi_{s}(i, k)+\sum_{k=0}^{W_{0}^{s}-1} \pi_{s}(0, k)_{e}=1$ we can calculate $\pi_{s}(0,0)$, hence, all the steady state probabilities.

Finally, since a node will make a transmission attempt in a given slot time if the Markov chain is in state $\pi_{s}(i, 0)$ for 


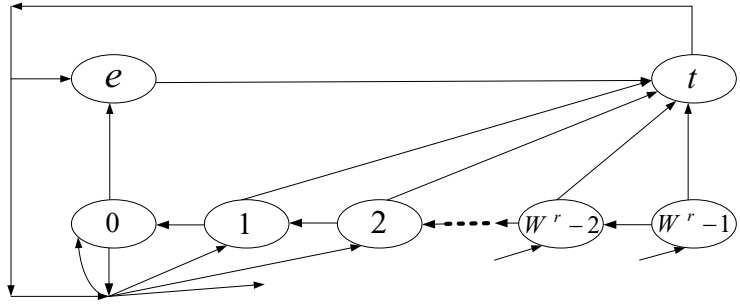

Fig. 3. Relay node's Markov model.

$i \in[0, m]$, then, $\tau_{s}$, the probability that a source node makes a transmission attempt in a given slot time can be expressed as $\tau_{s}=\sum_{i=0}^{m} \pi_{s}(i, 0)$.

2) Relay Node: Relay node operation is modeled using the Markov chain model of Fig. 3. The model has a single backoff stage represented by states $k \in\left[0, W^{r}-1\right]$. The backoff counter is uniformly chosen in that range, and the relay makes a transmission attempt when in state 0 . The relay node makes a transition to state $e$ if its queue becomes empty after a successful transmission. Finally, the chain is in state $t$ when the relay is attempting to transmit following a busy channel. Therefore

$$
\begin{array}{ll}
P\{k \mid 0\}=\frac{\left(1-q_{r}\right)\left(1-P_{f}^{r}\right)}{W^{r}}+\frac{P_{f}^{r}}{W^{r}}, & 0 \leq k \leq W^{r}-1 \\
P\{k \mid t\}=\frac{\left(1-q_{r}\right)\left(1-P_{f}^{\prime r}\right)}{W^{r}}+\frac{P_{f}^{\prime r}}{W^{r}}, & 0 \leq k \leq W^{r}-1
\end{array}
$$

where $q_{r}$ is the probability that a departing packet will leave the relay queue empty, $P_{f}^{r}$ is the probability of a failed relay transmission attempt out of state 0 , and $P_{f}^{\prime r}$ is the probability of a failed transmission attempt out of state $t$ (failure can be caused only by channel errors), and are given by $P_{f}^{r}=$ $1-\left(1-\tau_{s}\right)^{N}\left(1-P_{R A}^{\text {out }}\right)$ and $P_{f}^{\prime r}=\left(1-P_{R A}^{\text {out }}\right)$.

At the beginning of each idle slot time, the backoff counter is decremented, then $P\{k \mid k+1\}=P_{i}, \quad 0 \leq k \leq W^{r}-1$, where $P_{i}$ is the probability that the channel is sensed idle by the relay node (i.e., all $N$ source nodes are not attempting to transmit), and is given by $P_{i}=\left(1-\tau_{s}\right)^{N}$.

A successful transmission that leaves the relay queue empty leads to a transition to state $e$, then we have $P\{e \mid 0\}=q_{s}(1-$ $\left.P_{f}^{r}\right)$ and $P\{e \mid t\}=q_{s}\left(1-P_{f}^{\prime r}\right)$. Finally, transitions into state $t$ occur when the relay attempts to transmit a packet immediately after any transmission attempt on the channel, thus

$$
\begin{aligned}
& P\{t \mid e\}=N \tau_{s}\left(1-\tau_{s}\right)^{N-1} P_{s A P}^{\text {out }}\left(1-P_{s R}^{\text {out }}\right)=a \\
& P\{t \mid k\}=1-P_{i}, \quad 0<k \leq W^{r} .
\end{aligned}
$$

The rest of the analysis follows the same steps as in the case of the source nodes' chain, we will omit the rest of the analysis due to the lack of space.

\section{B. Queuing Analysis}

We assumed that each node receives packets from upper layer based on a Poisson arrival process with arrival rate $\lambda_{s}$ packets/sec, and fixed packet size $L$. Packet processing at each node can be seen as a single server with service rate $\mu_{s}$, which depends on the channel access mechanism, the interaction between different nodes, and the channel statistics. Therefore, node queues can be modeled as M/G/1 queues. In the case of the relay and for mathematical tractability, we will model the relay as an M/G/1 queuing system. Simulation results will later show that this is in fact a good approximation to the behavior of relay queue.

Since for any queueing system with single arrivals and departures, it holds that the queue length seen by an arriving customer is equal to its length left by a departure customer. Furthermore, using the PASTA (Poisson Arrivals See Time Averages) property [9] associated with Poisson arrivals, the queue length at an arbitrary time equals the queue length seen by an arriving customer. This enables us to calculate the probability that a node queue is empty as follows [9], $q_{s}=1-\frac{\lambda_{s}}{\mu_{s}}$, similarly, for the relay queue $q_{r}=1-\frac{\lambda_{r}}{\mu_{r}}$, which hold only when the queues are stable.

In the following we will calculate the service and arrival rates for the different network nodes.

1) Source Nodes Service Rate: Service time, $S_{s}$, of a packet is defined as the interval between the time the packet becomes at the head of the transmission queue and the time the packet is acknowledged for correct reception (reception by either the $\mathrm{AP}$ or the relay node). The probability generating function (PGF) [10] characterizing the distribution of the time spend at each counter step could now be written as

$$
\begin{aligned}
F(z) & =P_{i} z^{\sigma} \sum_{i=0}^{\infty} \sum_{j=0}^{i}\left(\begin{array}{l}
i \\
j
\end{array}\right)\left(P_{b 1} z^{T_{b}}\right)^{j}\left(P_{b 2} z^{2 T_{b}}\right)^{i-j} \\
& =\frac{P_{i} z^{\sigma}}{1-P_{b 1} z^{T_{b}}-P_{b 2} z^{2 T_{b}}},
\end{aligned}
$$

where $P_{b 1}=\pi_{r}(e)\left[1-\left(1-\tau_{s}\right)^{N-1}-(N-1) \tau_{s}(1-\right.$ $\left.\left.\tau_{s}\right)^{N-2} P_{s A}^{\text {out }}\left(1-P_{s R}^{\text {out }}\right)\right]$ is the probability that the relay does not access the channel immediately after a source node transmission, thus a busy period encountered by the node is of duration $T_{b}$. Busy periods of duration $2 T_{b}$ occur if the relay accesses the channel immediately after a source node transmission which has a probability probability $P_{b 2}=\pi_{r}(e)(N-1) \tau_{s}\left(1-\tau_{s}\right)^{N-2} P_{s A}^{\text {out }}\left(1-P_{s R}^{\text {out }}\right)+$ $\left[1-\left(1-\tau_{s}\right)^{N-1}\right] \sum_{k=1}^{W^{r}-1} \pi_{r}(k)$. The distribution of the time spent in stage $i$ is characterized by the PGF $F_{i}(z)=$ $\sum_{k=0}^{W_{i}^{s}-1} \frac{F^{k}(z)}{W_{i}^{s}}$. And finally the PGF for the service time $S_{s}$ can be written as

$$
\begin{aligned}
G_{s}(z)= & \left(1-P_{f}^{s}\right) z^{T_{b}}\left[\sum_{i=0}^{m-1}\left(P_{f}^{s} z^{T_{b}}\right)^{i} \prod_{j=0}^{i} F_{j}(z)\right. \\
& \left.+\frac{\left(P_{f}^{s} z^{T_{b}}\right)^{m} \prod_{j=0}^{m} F_{j}(z)}{1-P_{f}^{s} z^{T_{b}} F_{m}^{i}(z)}\right],
\end{aligned}
$$

which accounts for the busy slot in which the packet is successfully delivered, the possible number of failures a packet encounters (hence, the number of backoff stages it goes through), and finally, the amount of time probably spent at 
the maximum backoff stage $m$. The service rate can then be calculated by differentiating $G_{s}(z)$ and setting $z=1$, $\mu_{s}^{-1}=E\left[S_{s}\right]=\left.\frac{d G_{s}(z)}{d z}\right|_{z=1}$.

2) Relay Node Arrival Rate: The time, $A_{r}$, between packet arrivals to the relay queue is composed of the following components: (i) Idle periods in which no node (source or relay) is transmitting. This periods have a length $\sigma$ and probability $P_{i}=\left(1-\tau_{s}\right)^{N}\left(1-\tau_{r}\right)$. (ii) Busy periods of duration $T_{b}$, which occur if the relay queue is empty and the transmission attempt does not result in an arrival at the relay. This occurs with probability $P_{b 1}=\pi_{r}(e)\left[1-\left(1-\tau_{s}\right)^{N-1}-(N-1) \tau_{s}(1-\right.$ $\left.\left.\tau_{s}\right)^{N-2} P_{s A}^{\text {out }}\left(1-P_{s R}^{\text {out }}\right)\right]$. (iii) Busy periods of duration $2 T_{b}$ not resulting in a relay arrival, which occur if the relay queue is not empty when a source node makes a transmission attempt. Thus has a probability $P_{b 2}=\left[1-\left(1-\tau_{s}\right)^{N-1}-(N-\right.$ 1) $\left.\tau_{s}\left(1-\tau_{s}\right)^{N-2} P_{s A}^{\text {out }}\left(1-P_{s R}^{o u t}\right)\right] \sum_{k=1}^{W^{r}-1} \pi_{r}(k)$. (iv) Finally, a busy period during which a packet enters the relay queue. This will always have a duration $T_{b}$, and has a probability $P_{a}=$ $\left[\pi_{r}(e)+\sum_{k=1}^{W^{r}-1} \pi_{r}(k)\right](N-1) \tau_{s}\left(1-\tau_{s}\right)^{N-2} P_{s A}^{\text {out }}\left(1-P_{s R}^{\text {out }}\right)$. The PGF of $A_{r}$ can then be written as,

$$
\begin{aligned}
G_{a}(z)= & P_{a} z^{T_{b}} \sum_{i=1}^{\infty} \sum_{j=1}^{i} \sum_{k=0}^{i-j}\left[\frac{i !}{j ! k !(i-j-k) !}\right. \\
& \left.\cdot\left(P_{i} z^{\sigma}\right)^{j}\left(P_{b 1} z^{T_{b}}\right)^{k}\left(P_{b 2} z^{2 T_{b}}\right)^{i-j-k}\right] .
\end{aligned}
$$

The arrival rate can then be calculated by differentiating $G_{a}(z)$ and setting $z=1, \lambda_{r}^{-1}=E\left[A_{r}\right]=\left.\frac{d G_{a}(z)}{d z}\right|_{z=1}$.

3) Relay Node Service Rate: Similar reasoning leads to the following expressions for PGF of the relay's service time $S_{r}$,

$$
\begin{aligned}
G_{r}(z)= & \pi_{r}(e) a\left(1-P_{f}^{\prime r}\right) z^{T_{b}}+\left(a P_{f}^{\prime r} z^{T_{b}}+\sum_{k=0}^{W^{r}-1} \pi_{r}(k)\right) \\
& \cdot\left[\left(F_{0}(z)\left(1-P_{f}^{r}\right) z^{T_{b}}+F_{t}(z)\left(1-P_{f}^{\prime r}\right) z^{T_{b}}\right)\right. \\
& \left.\cdot \sum_{i=0}^{\infty} \sum_{j=0}^{i}\left(\begin{array}{l}
i \\
j
\end{array}\right)\left(F_{0}(z) P_{f}^{r} z^{T_{b}}\right)^{j}\left(F_{t}(z) P_{f}^{\prime r} z^{T_{b}}\right)^{i-j}\right],
\end{aligned}
$$

where $F_{0}=\sum_{k=0}^{W^{r}-1} \frac{P_{i}^{k} z^{k \sigma}}{W^{r}}$ is the PGF characterizing the distribution of the time the packet at the head of the queue spends in the backoff stage before the backoff counter reaches 0. $F_{t}=\left(1-P_{i}\right) z^{T_{b}} \sum_{k=0}^{W^{r}-2} \frac{P_{i}^{k} z^{k \sigma}}{W^{r}}$ is the PGF of the time the packet at the head of the queue spends in the backoff stage before the Markov chain reaches state $t$ (composed of a single busy period and a maximum of $W^{r}-1$ idle slots). And $a=$ $N \tau_{n}\left(1-\tau_{n}\right)^{N-1} P_{s A}^{o u t}\left(1-P_{s R}^{o u t}\right)$ is the probability that a packet enters relay queue. Finally, $\mu_{r}^{-1}=E\left[S_{r}\right]=\left.\frac{d G_{r}(z)}{d z}\right|_{z=1}$.

\section{Network Throughput}

Normalized network throughput is defined as the fraction of time the channel is used to successfully transmit payload bits to the AP can be expressed as $S=\frac{P_{s} \cdot T_{p}}{T_{s}}$ where $P_{s}$ is the probability of a successful transmission to the AP (by source or relay nodes), $T_{p}$ is the time to transmit the payload part of a packet, and $T_{s}$ is the expected slot duration.

Four different events lead to a successful packet delivery to the AP: (i) packet is delivered directly through the source node or through immediate relay help, this has a probability $P_{s}^{1}=\pi_{r}(e)\left[N \tau_{s}\left(1-\tau_{s}\right)^{N-1}\left(\left(1-P_{s A}^{\text {out }}\right)+P_{s A}^{\text {out }}\left(1-P_{s R}^{\text {out }}\right)(1-\right.\right.$ $\left.\left.\left.P_{R A}^{o u t}\right)\right)\right]$. (ii) Relay delivers the packet at the head of its queue after a source node's packet failure, this occurs with probability $P_{s}^{2}=\left[1-\left(1-\tau_{s}\right)^{N}-N \tau_{s}\left(1-\tau_{s}\right)^{N-1}(1-\right.$ $\left.\left.P_{s A}^{o u t}\right)\right] \sum_{k=0}^{W^{r}} \pi_{r}(k)$. (iii) The relay and a source node delivers two successful packets to the AP, this occurs with probability $P_{s}^{3}=N \tau_{s}\left(1-\tau_{s}\right)^{N-1}\left(1-P_{s A}^{\text {out }}\right)\left(1-P_{R A}^{\text {out }}\right) \sum_{k=0}^{W^{r}} \pi_{r}(k)$. (iv) The relay succeeds in transmitting a packet when its backoff counter reaches 0 , which has a probability $P_{s}^{4}=$ $\tau_{r}\left(1-\tau_{s}\right)^{N}\left(1-P_{R A}^{o u t}\right)$. Finally, the probability $P_{s}=P_{s}^{1}+$ $P_{s}^{2}+2 P_{s}^{3}+P_{s}^{4}$. The factor of 2 before $P_{s}^{3}$ accounts for the fact that the associated event results in the successful delivery of two packets to the AP.

The average length of a randomly chosen slot time is given by $T_{s}=\left(1-\tau_{s}\right)^{N}\left(1-\tau_{r}\right) \sigma+\left[\tau_{r}+\pi_{r}(e)\left(1-\left(1-\tau_{s}\right)^{N}-\right.\right.$ $\left.\left.N \tau_{s}\left(1-\tau_{s}\right)^{N-1} P_{s A}^{\text {out }}\left(1-P_{s R}^{\text {out }}\right)\right)\right] T_{b}$

$+2 \pi_{r}(t) T_{b}$, which accounts for the idle slots, busy slots in which relay transmits or a source node transmission is not followed by relay transmission (when relay queue is empty and no arrivals occur during source transmission), and busy slots in which a relay transmission follows source transmissions.

Based on an $11 \mathrm{Mbps}$ transmission rate, and payload of length $L=2312$ octets, typical slot duration are $\sigma=20 \mu \mathrm{s}$, $T_{p}=1681.5 \mu \mathrm{s}$, and $T_{b}=2160.4 \mu \mathrm{s}$.

\section{RESULTS AND DISCUSSIONS}

We compare the performance of the cooperative protocol and the CSMA/CA protocol without cooperation. We set the SNR threshold $\beta=15 \mathrm{~dB}$ and the path loss exponent $\gamma=3.7$. The distance between any node and AP is $120 \mathrm{~m}$, and between any node and the relay $70 \mathrm{~m}$, and between relay and AP 50 $\mathrm{m}$. Transmission power is $100 \mathrm{~mW}$, and noise variance $N_{0}=$ $10^{-11}$. Source node's initial contention window $W_{0}^{s}=32$ with $m=5$ backoff stages, and relay node's contention window size $W_{0}^{r}=32$.

Fig. 4 depicts the maximum aggregate arrival rate supported by the network while maintaining queues stability versus the number of network nodes. We can observe that, for a given number of nodes, the proposed cooperative protocols resulted in a $7 \%$ average increase in the maximum supported aggregate arrival rate. This increase is due to the fact that the relay node provides a more reliable path to the AP leading to a higher packet delivery rate. Therefore, source nodes are able to empty their queues at a faster rate, thus, freeing the channel for relay access, and for additional nodes that the network might accommodate.

The normalized network throughput is depicted in Fig. 5 as a function of the number of network nodes for a fixed arrival rate of $\lambda_{s}$. The results shown are under the condition that all the network queues are stable. It is noted that the network throughput is almost identical under both the cooperative 


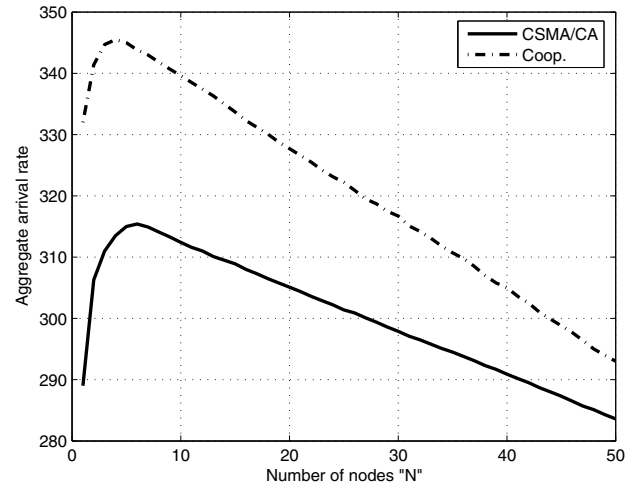

Fig. 4. Maximum achievable aggregate arrival rate vs number network nodes.

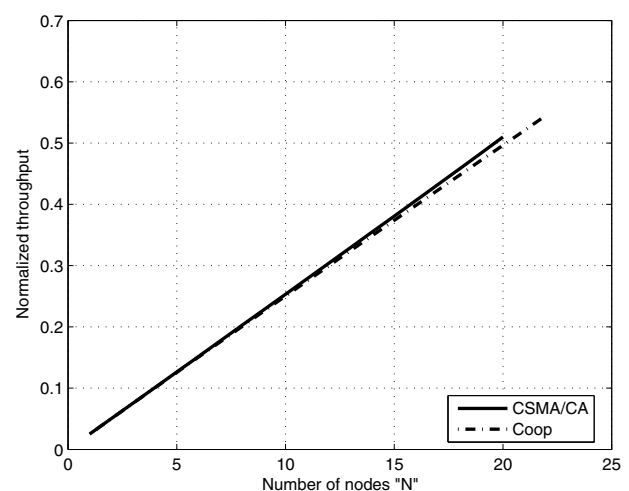

Fig. 5. Normalized throughput vs. number of nodes for $\lambda_{s}=15$.

and non-cooperative scenarios. This can be interpreted as follows; for a stable queue, it is well known that the average departure rate from the queue is equal to its average arrival rate. Therefore, for a given arrival rate, the average number of successfully transmitted packets to the AP will always be the same under both cooperative and non-cooperative protocols, as long as the queues are stable. On the other hand, the number of nodes supported by the network has increased by about $10 \%$.

Fig. 6 compares between the collision probability of CSMA/CA and our cooperative protocol. We notice a decrease in the collision probability, which is because of the second path to the AP the relay offers to the network nodes. This second path helps the different nodes empty their queues at a faster rate, hence, nodes do not have to access the channel as often as in the case without cooperation, which reduces the collision probability.

\section{CONCLUSIONS}

A novel cooperative protocol for IEEE 802.11 based wireless random access networks is developed in this paper. Cooperation is achieved through a relay node that will helps different network nodes forward their packets to the AP without the penalty of increased collision probability, which

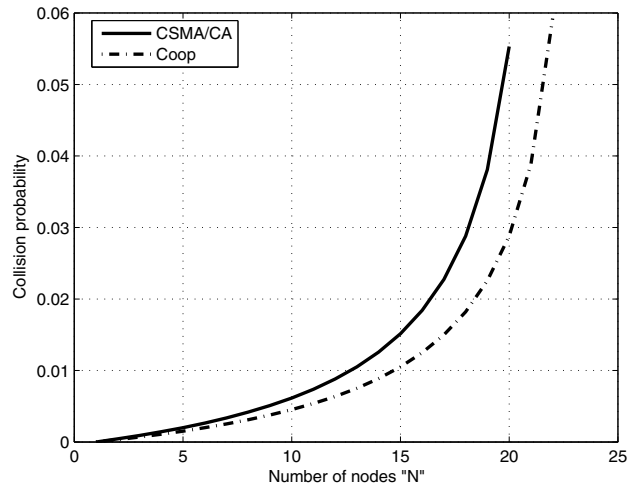

Fig. 6. Collision probability vs. number of nodes for $\lambda_{s}=15$.

is common in random access protocols when additional nodes are added to the network.

The performance of the proposed cooperation protocol is characterized through the development of a Markov model coupled with queuing analysis of the network operation. The developed Markov model accurately described the network dynamics in the presence of relay, and captured the interactions between different network nodes. Results revealed a significant improvement in terms of the maximum achievable arrival nodes's rates, and the number of nodes supported by the network.

\section{REFERENCES}

[1] A. K. Sadek, K. J. R. Liu, and A. Epheremides, "Cognitive multiple access via cooperation: Protocol design and performance analysis," IEEE Trans. on Information Theory, vol. 53, no. 10, pp. 3677-3696, Oct. 2007.

[2] A. A. El-Sherif, A. Kwasinski, A. K. Sadek, and K. J. R. Liu, "Content-aware multiple access protocol for cooperative packet speech communications," IEEE Trans. on Wireless Communications, vol. 8, no. 2, pp. 995-1005, Feb. 2009.

[3] R. Lin and A. P. Petropulu, "A new wireless medium access protocol based on cooperation," IEEE Trans. Signal Processing, vol. 52, no. 12, pp. 4675-4684, Dec. 2005.

[4] M. K. Tsatsanis, R. Zhang, , and S. Banerjee, "Network-assisted diversity for random access wireless networks," IEEE Trans. on Signal Processing, vol. 48, pp. 702-711, March 2000.

[5] IEEE Computer Society LAN MAN Standards Committee, Wireless LAN medium access control (MAC) and physical layer (PHY) specifications, IEEE Standard 802.11-1999, New York, NY: IEEE, 1999.

[6] Z. Hadzi-Velkov and B. Spasenovski, "On the capacity of ieee 802.11 dcf with capture in multipath-faded channels," in Proc. Intl. Conf. on Comm. (ICC), Jul. 2003, pp. 121-126.

[7] Y. Zheng, K. Lu, D. Wu, and Y. Fang, "Performance analysis of ieee 802.11 dcf in imperfect channels," vol. 55, no. 5, pp. 1648-1656, Sep. 2006.

[8] D. Malone, K. Duffy, and D. Leith, "Modelling the 802.11 distributed coordination function in nonsaturated heterogeneous conditions," vol. 15, no. 1, pp. 159-172, Feb. 2007.

[9] Ronald W. Wolff, Stochastic Modeling and the Theory of Queues, Prentice Hall, 1989.

[10] G. R. Grimmett and D. R. Stirzaker, Probability and Random Processes, Oxford University Press, 2001. 